\title{
Impact of formal tuition, supervised practice and feedback on the acquisition of basic laparoscopic skills by novices using a box trainer
}

\section{Mathew Lyons, Oliver Brown and Brian Ip*}

University of Edinburgh Medical School, 51 Little France Crescent, Edinburgh, EH16 4SA, United Kingdom

${ }^{*}$ Corresponding author at: Dumfries Royal Infirmary, Cargenbridge, Dumfries, DG2 8RX, United Kingdom.

Email: brian.ip@nhs.scot

Date accepted for publication: 25 March 2021

\section{Abstract}

Background and Aim: Laparoscopic box trainers are valuable in the teaching of basic laparoscopic skills. This study aims to define the impact of formal tuition, supervised practice and feedback on the acquisition of basic laparoscopic skills on a box trainer by novice. Methods: All medical undergraduate attendees of a conference were invited to participate. Participants with previous experience on laparoscopic simulators were excluded. Twenty-eight were randomized to the control group (self-directed learning/practice), 23 to the intervention group (formal tuition/supervised practice/feedback). Baseline and post-intervention performance were assessed using the Global Operative Assessment of Laparoscopic Skills (GOALS) score by blinded assessors. Results: Both groups showed an overall increase in GOALS score after exposure to the box trainer: control group, 7.79 (SD, 2.23) pre-intervention versus 8.25 (SD 3.04) postintervention; intervention group: 8.43 (SD 2.13) pre-intervention versus 9.35 (SD 2.42) post-intervention $(P=0.52)$. The intervention group showed a greater overall increase in GOALS score compared with the control group, but this was not statistically significant (control mean improvement, +0.46 [SD 4.08] versus intervention mean improvement +0.91 [SD 3.84]; $P=0.66$ ). A lower performance in depth perception was reported for the control group (mean, -0.07 ). Conclusions: Box trainers are effective in assisting novices to acquire basic laparoscopic skills. Expert tuition, supervised practice and feedback appear to have an additional positive effect.

Keywords: laparoscopy; simulation; teaching; practice; feedback; GOALS

\section{Introduction}

Today's surgical trainees face many challenges in acquiring technical competencies. Contributing factors include an overall reduction in working hours, ${ }^{1}$ a shift from a single mentor to multiple trainers, ${ }^{2}$ pressure on practicing surgeons and health care organizations to deliver services, and the increasing use of non-operative management for selected conditions (e.g. appendicitis). ${ }^{3}$ The recent COVID-19 pandemic has created even more barriers to training. These barriers include cancellation of elective surgery, reduction in primary care referrals, staff redeployment and self-isolation., ${ }^{4,5}$ Some professional guidelines caution on the routine use of laparoscopy at the height of the pandemic due to the theoretical risk of aerosol transmission, ${ }^{6,7}$ further reducing exposure to laparoscopic skills.

Simulation-based training has been shown to promote skills transfer to the real-life operative environment. ${ }^{8}$ In laparoscopic surgery, a selection of simulators have been trialled and received positive feedback. ${ }^{9}$ One type of simulator is the box trainer..$^{10}$ Due to its portability and ease of use, trainees can train on demand in the safety of their homes. Although numerous studies have assessed the impact of box trainers in improving performance, ${ }^{10}$ there is less evidence evaluating the combined impact of expert tuition, supervised practice and feedback on simulator performance. One prospective randomized controlled study demonstrates better simulator performance with supervision and feedback, although the study population in each arm was small $(n=5) .{ }^{11}$ As a result, there is a concern that inappropriate use of box trainers without formal tuition and feedback can lead to acquisition of poor techniques by novices, leading to the need for future remedial correction.

Feedback is most effective when delivered in the right format and at the right time. Effective feedback can 
reinforce good behaviour and reduce errors. It increases confidence in the trainee through the establishment of familiarity and rapport between the trainee, environment and trainer. Skills are more likely to be retained in the long term. In the pandemic era, effective feedback in simulated settings helps teams cope with the stress and uncertainty of working with new protocols. ${ }^{12}$ Feedback can vary by source (oral/written), type (formative/summative), approach (active/passive), facilitator (expert/non-expert), and timing (concurrent/frequency/duration). ${ }^{13}$

We hypothesize that formal tuition and feedback has no impact on the acquisition of basic laparoscopic skills by novices using the box trainer. A single-blind randomized controlled trial was designed to establish whether formal tuition, supervised practice and feedback has any advantage compared with self-directed practice on improving task performance of basic laparoscopic tasks by novices on box trainers.

\section{Material and methods}

All medical undergraduates who registered and attended a 1-day surgical conference (March 2016, Royal College of Surgeons of Edinburgh) were invited to participate in the study. Informed consent was obtained from all participants. Participant demographics were collected by questionnaire. Participants with any previous experience of using portable laparoscopic simulators were excluded.

Following an agreed study protocol, the cohort was randomized to self-directed learning and practice on the simulator (control group) versus structured tuition, supervised practice and feedback (intervention group) (Fig. 1). Block randomization was carried out by a dedicated college administrator. A modified Global Operative Assessment of Laparoscopic Skills (GOALS) scoring system ${ }^{14}$ was used to assess the baseline and post-intervention performance of two basic tasks on eoSim Laparoscopic Simulators (eoSurgical, Edinburgh, UK; Fig. 2). ${ }^{15}$ GOALS was used because of its ease of use, validity and reliability. The tissue handling domain of GOALS was excluded because no soft tissue was used for the exercises. For logistical purposes, participants were divided into four consecutive workshops; two workshops were dedicated to the control group and two to the intervention group.

Assessment of baseline and post-intervention laparoscopic tasks was conducted by six trained assessors. All assessors were given verbal and written training before the start of the trial on objective assessments of the participants. Baseline assessment assessed the participant's ability to stack ringshaped mints (Polo Mints) on a vertical stick for a $2 \mathrm{~min}$ or until all mints had been stacked. Post-intervention assessment tested the participant's ability to thread a shoelace through a series of loops. A plastic board with five loops on 2-cm high stalks was placed in the field of view of the camera. Participants were asked to thread a shoelace in an S-shaped pattern through the loops in a maximum time of 2 min. To minimize disadvantage of handedness, those who were right hand dominant completed the $\mathrm{S}$ pattern from right to left and those with left hand dominance completed the task from left to right. Different baseline and post-intervention tasks were selected to mitigate the confounding effect of repetitive practice on observed performance.

In the control group, participants were given $20 \mathrm{~min}$ to read written instructions and practice stacking the ring-shaped mints independently on the box trainers. During this time, the control group worked at their own pace to balance learning and practice. After $20 \mathrm{~min}$, the control group underwent the final assessment. In the intervention group, participants were provided with a 10 -min didactic tutorial by a senior surgical trainee. The tutorial introduced the functions and correct usage of each laparoscopic instrument, as well as instructions on achieving the best ergonomics for stacking the ring-shaped mints. Participants then spent $10 \mathrm{~min}$ practicing stacking the ring-shaped mints on box trainers, with direct supervision from surgical trainees at a participant/tutor ratio of 2:1. Individual formative feedback was given to the intervention group throughout and after practice. The intervention group then underwent the final assessment. Final assessment for both groups was carried out by assessors blinded to the type of intervention. Statistical analysis of baseline and post-intervention GOALS scores was carried out using MatLab (MathWorks, Natick, MA, USA) and SPSS (IBM Corp., Armonk, NY, USA). Shapiro-Wilk tests were used to confirm normality. Independent sample $t$ tests and one-way ANOVA were used to determine statistical differences at the $5 \%$ significance level.

\section{Results}

Fifty-five medical undergraduates registered and attended the 1-day surgical conference. All consented to participate in this study. Twenty-seven were assigned to the intervention group and 28 were assigned to the control group (Fig. 1). Four participants in the intervention group failed to attend the scheduled lecture and supervised practice, leaving 23 participants for the final analysis. Participant demographics are shown in Table 1. There were no statistical differences with regard to sex, level of seniority, and handedness. All participants were aged between 18 and 27 years. 


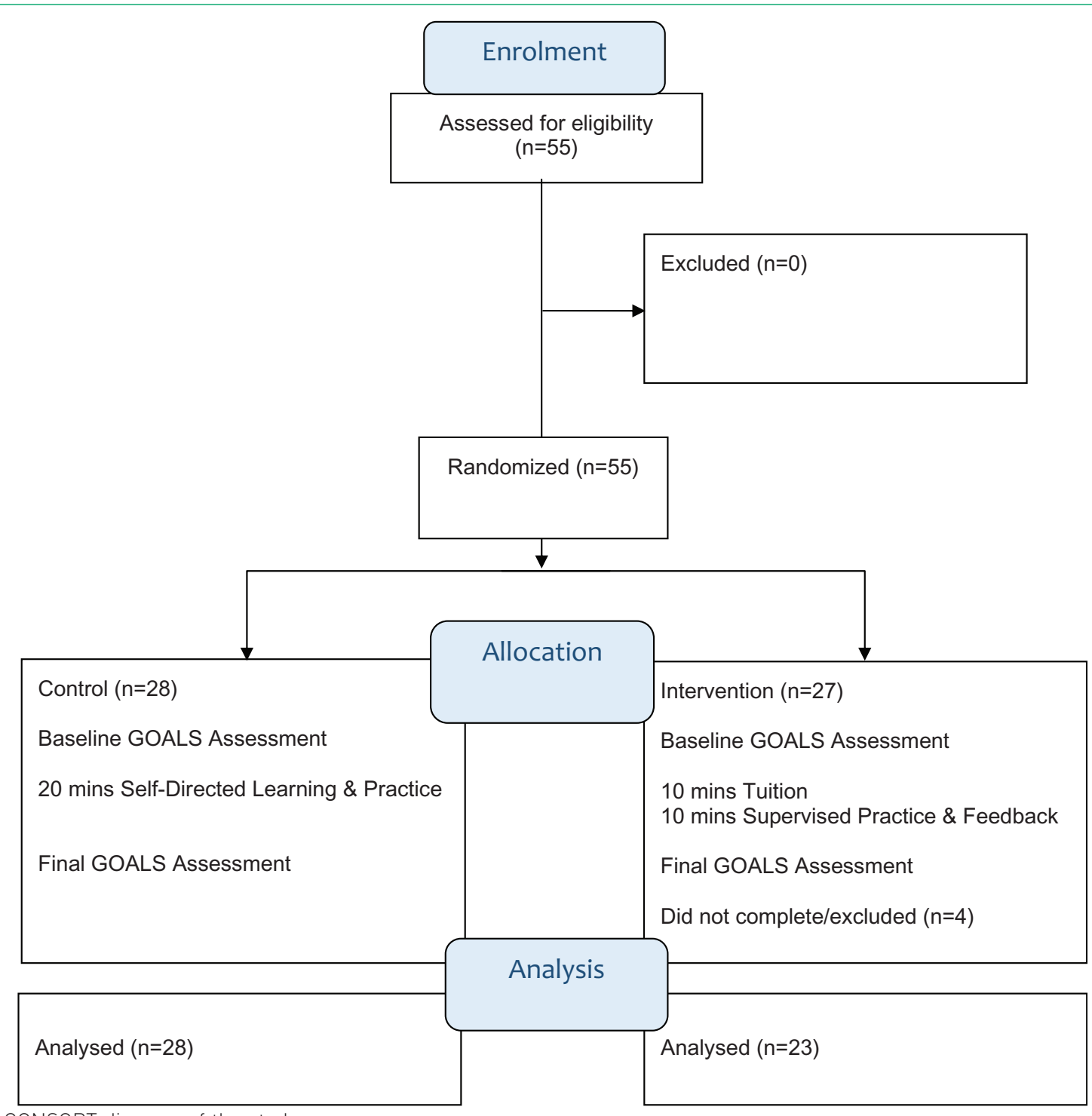

Figure 1. CONSORT diagram of the study.

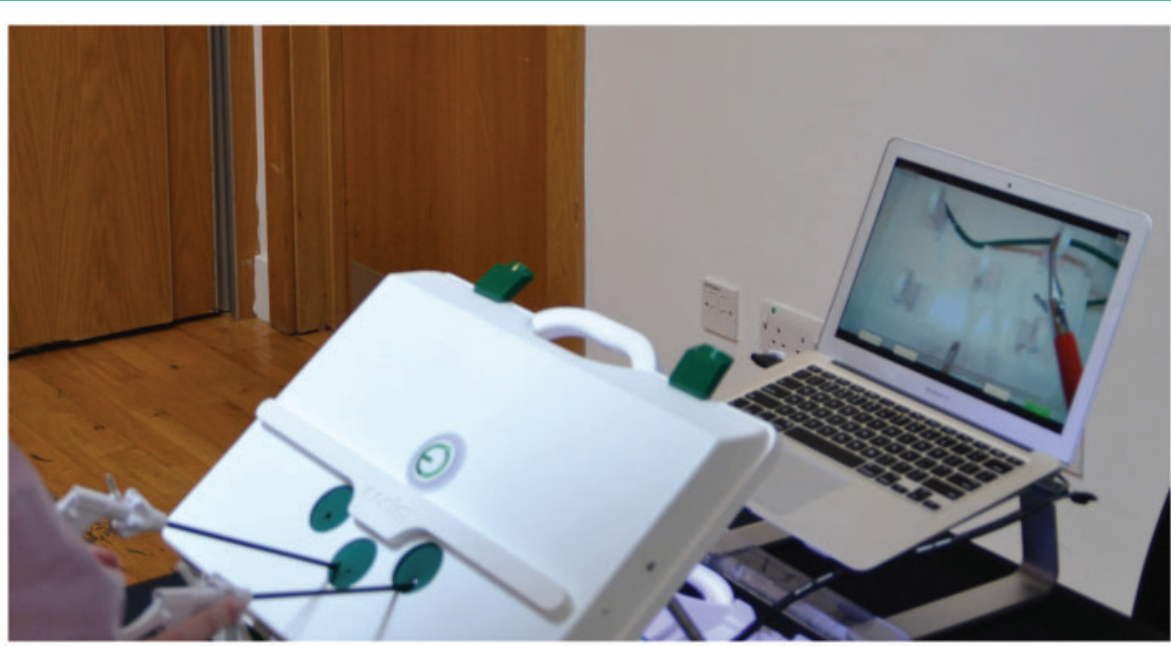

Figure 2. eoSim laparoscopic box trainer with final assessment (threading a shoelace) shown on the computer screen. 
Overall GOALS scores recorded by individual tutors in the baseline and post-intervention assessments were similar ( $P=0.92$ and $P=0.43$, respectively; Fig. 3 ), suggesting good reliability of GOALS as an assessment tool. Overall baseline GOALS scores were similar between the control and intervention groups ( $7.79 \pm 2.23$ vs $8.43 \pm 2.13$; $P=0.30$; Fig. 4 and Table 2). There was no statistical difference in the individual GOALS domains between the control and intervention groups $(2.86 \pm 0.93$ vs $3.0 \pm 1, P=0.60$ for depth perception, $2.21 \pm 0.96$ vs $2.43 \pm 0.90, P=0.40$ for bimanual dexterity; $2.71 \pm 0.9$ vs $3.0 \pm 0.8, P=0.24$ for efficiency; Table 2).

Table 1. Participant demographics

\begin{tabular}{llll}
\hline & $\begin{array}{l}\text { Control group } \\
(\boldsymbol{n}=\mathbf{2 8})\end{array}$ & $\begin{array}{l}\text { Intervention } \\
\text { group }(\boldsymbol{n}=\mathbf{2 3})\end{array}$ & $\boldsymbol{P}$ \\
\hline $\begin{array}{l}\text { Male:female ratio } \\
\text { Seniority of participant }\end{array}$ & $13: 15$ & $15: 8$ & 0.23 \\
Year 1 & 4 & 2 & \\
Year 2 & 8 & 6 & \\
Year 3 & 2 & 2 & \\
Year 4 & 10 & 7 & NS \\
Year 5 & 3 & 3 & \\
Year 6 & 1 & 3 & \\
Right hand dominant & $27 / 28$ & $23 / 23$ & \\
\hline
\end{tabular}

Post-intervention overall GOALS scores were also similar between the control and intervention groups, indicating no advantage for either group $(8.25 \pm 3.04$ vs $9.35 \pm$ 2.42, $P=0.17$; Fig. 4 and Table 2). This observation is consistent between the GOALS sub-domains $(2.79 \pm 1.13$ vs $3.04 \pm 0.88, P=0.37$ for depth perception; $2.71 \pm 1.08$ vs $3.17 \pm 0.98, P=0.12$ for bimanual dexterity; $2.75 \pm 1$ vs $3.13 \pm 0.87, P=0.16$ for efficiency; Table 2).

Overall GOALS score increased from baseline in both groups (control group: $+0.46 \pm 4.08$ intervention group, $+0.91 \pm 3.84, P=0.17$; Table 3). Although a greater increase was observed across all GOALS domains in the intervention group, there was no statistical difference when compared with the control group. In the intervention group, the lowest improvement was reported for depth perception (mean, $+0.044 \pm 1.61$ ). Paradoxically, depth perception was poorer after self-directed learning and practice in the control group (mean, $-0.7 \pm 1.63$ ).

\section{Discussion}

Laparoscopic box trainers have previously been shown to reduce the time to task completion, reduce errors, and improve overall performance. ${ }^{16}$ The impact of tuition, supervised practice and feedback on box trainer users is less formally quantified. One observational study showed an improvement in GOALS scores for surgical trainees who used a box trainer in conjunction with a structured

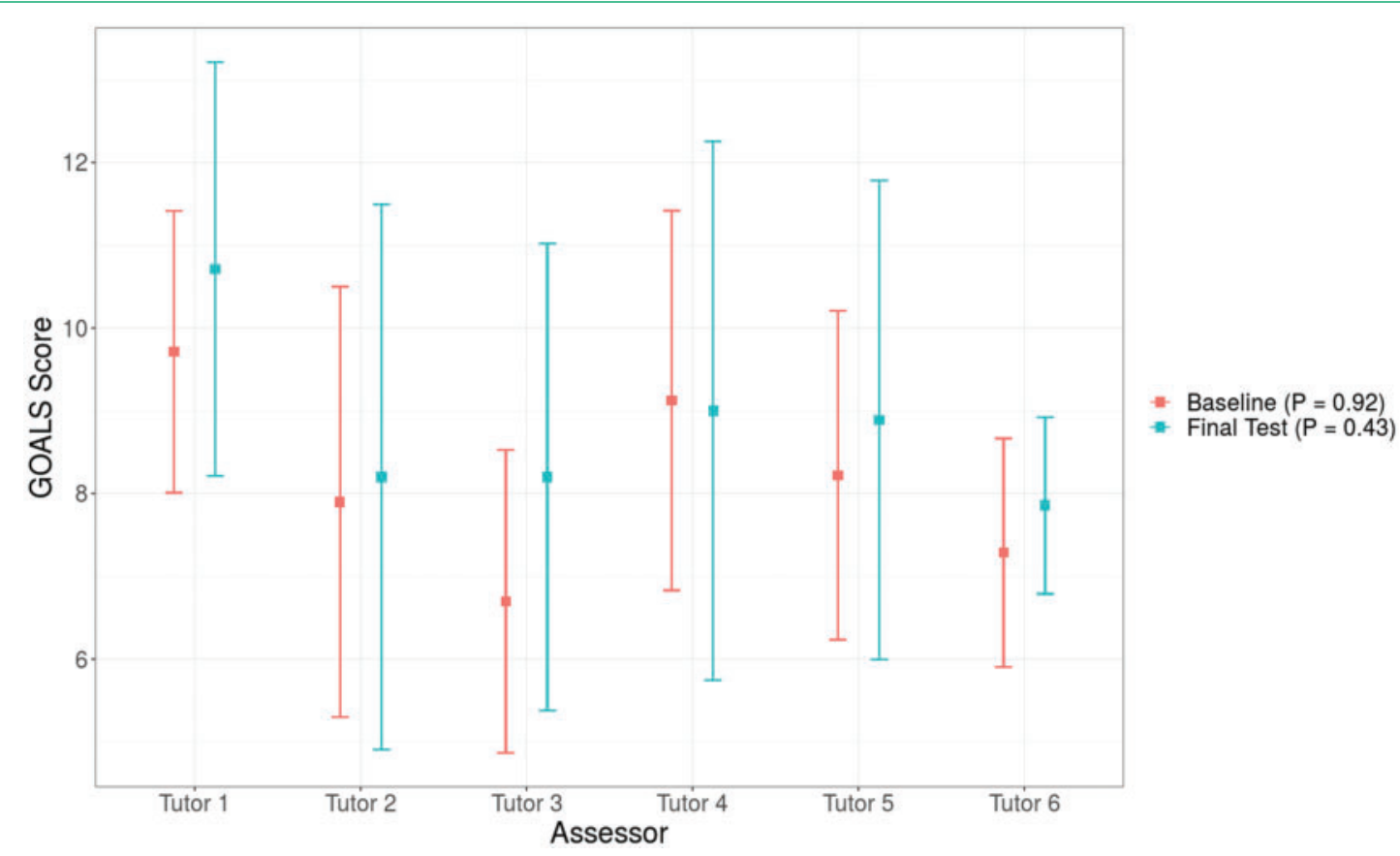

Figure 3. Comparison of baseline and post-intervention GOALS scores between assessors. 
Table 2. Comparison of GOALS scores before and after the intervention

\begin{tabular}{|c|c|c|c|c|c|c|}
\hline \multirow[t]{2}{*}{ Measure } & \multicolumn{3}{|l|}{ Pre-intervention assessment } & \multicolumn{3}{|l|}{ Post-intervention assessment } \\
\hline & Self-directed mean score (SD) & Tutorial mean score (SD) & $P$ & Self-directed mean score (SD) & Tutorial mean score (SD) & $P$ \\
\hline GOALS & $7.79(2.23)$ & $8.43(2.13)$ & 0.30 & $8.25(3.04)$ & $9.35(2.42)$ & 0.17 \\
\hline Depth perception & $2.86(0.93)$ & $3.0(1)$ & 0.60 & $2.79(1.13)$ & $3.04(0.88)$ & 0.37 \\
\hline Bimanual dexterity & $2.21(0.96)$ & $2.43(0.9)$ & 0.40 & $2.71(1.08)$ & $3.17(0.98)$ & 0.12 \\
\hline Efficiency & $2.71(0.9)$ & $3.0(0.8)$ & 0.24 & $2.75(1.00)$ & $3.13(0.87)$ & 0.16 \\
\hline
\end{tabular}

Table 3. Overall mean improvement

\begin{tabular}{llll}
\hline Measure & $\begin{array}{l}\text { Control group } \\
\text { mean } \\
\text { improvement (SD) }\end{array}$ & $\begin{array}{l}\text { Intervention } \\
\text { group mean } \\
\text { improvement (SD) }\end{array}$ & $\boldsymbol{P}$ \\
\hline GOALS & $+0.46(4.08)$ & $+0.91(3.84)$ & 0.66 \\
Depth perception & $-0.07(1.63)$ & $+0.044(1.61)$ & 0.77 \\
Bimanual dexterity & $+0.5(1.43)$ & $+0.74(1.39)$ & 0.54 \\
Efficiency & $+0.036(1.45)$ & $+0.13(1.39)$ & 0.8 \\
\hline
\end{tabular}

curriculum versus those who used a box trainer without a structured curriculum. ${ }^{17}$ In the context of other (non-box trainer) laparoscopic simulators, there is evidence that feedback given at the end of a task can improve task performance. $^{18,19}$ There does not appear to be any significant difference between feedback given by peers versus experts on a newly taught basic technical task. ${ }^{20}$ Expert tuition on these simulators appears to have limited effect. ${ }^{21,22}$

This study attempts to examine the impact of short intensive tuition, supervised practice and feedback on the acquisition of basic laparoscopic skills in the early phase of learning. The authors hypothesized that learning is too robust at this early formative phase for the novice for tuition to exert maximum benefit. However, on the contrary, our study has demonstrated that formal tuition, feedback and practice may deliver improvement over self-directed learning and practice alone across all GOALS domains, although this is not statistically significant. The lack of significant performance gains is invariably due to the short time lapse between the intervention and final assessment. It is postulated that a longer time lapse ranging from several hours to days would allow for adequate rest, reflection and consolidation.

A different and more complex task was deliberately chosen as the post-intervention assessment. The rational for this was to mitigate repetitive practice as a confounding factor in affecting the observed performance. Given that it takes a minimum of 45 min to acquire a new basic technical skill, we do not think the practice component had a significant influence on the observations. ${ }^{23-25}$

Both groups improved after short exposure to the portable simulator, further supporting its use in simulation. The only sub-domain showing very minimal improvement or apparent regression was depth perception. In laparoscopic surgery, binocular disparity is not freely available. Operators are required to mentally rebuild a three-dimensional picture of the task. An extended period of training and compensation is needed for competency in this domain to be achieved. Therefore our findings are expected and in keeping with current knowledge. ${ }^{26}$

This study has several limitations. It was not realistic to perform a power calculation, because the final number of trial participants was dictated by the availability of conference attendees on the day. Tuition and supervised feedback were given in a short period of time and on one occasion, meaning the learning curve was not fully realized. Only positive performance was recorded. The observation and recording of errors were felt to be too resource consuming in this study. Errors also need to pre-defined for each exercise to avoid observer bias. Baseline and final assessment exercises were limited to $2 \mathrm{~min}$ each due to the confines of a tight working timetable. A longer period of observation may produce more reliable assessments.

At the time of writing, COVID-19 continues to have a detrimental impact on surgical training, resulting in a significant decline in all operations and contact with trainers. With COVID-19 and its variants likely to remain prevalent indefinitely, attention should now focus on how surgical education and training should be adapted and delivered in a controlled COVID-19 environment. To make up the shortfall in live operating and supervised simulated training, many surgical trainees are resorting to unsupervised training on box trainers. Lack of feedback could mean the emergence of non-validated or non-ergonomic techniques, 


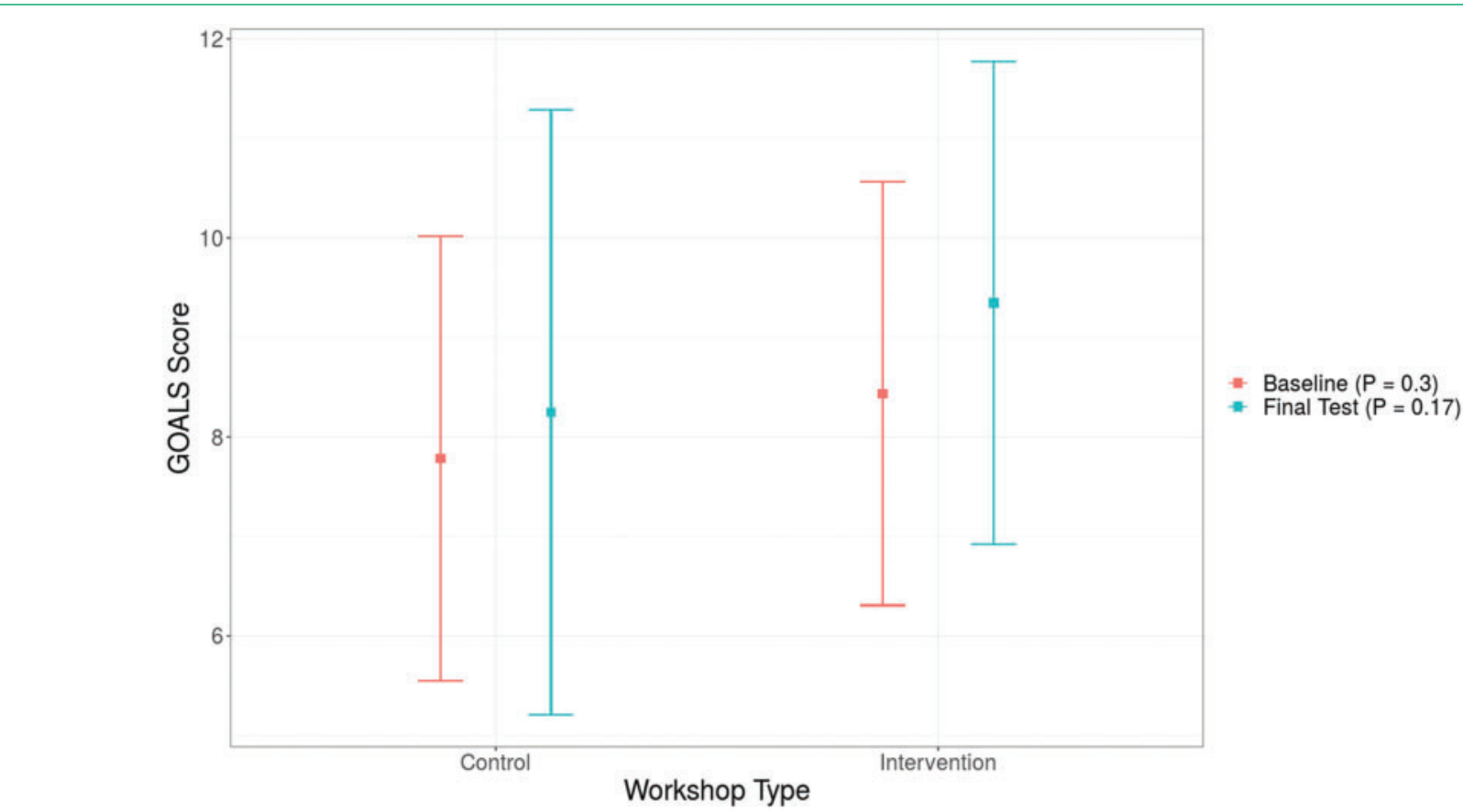

Figure 4. Comparison of mean GOALS scores between the control and intervention groups.

requiring time-consuming remedial correction at a later stage. Trainees who have fewer distractions at home and those who have higher motivation and self-awareness in isolation will be more likely to succeed. In the United States, the Fundamentals of Laparoscopic Surgery Programme provides a structured learning approach using selected clinical scenarios. ${ }^{27}$ Artificial intelligence, through its analysis of structured and unstructured data (e.g. reflection diaries, video), may provide even more detailed feedback. ${ }^{28}$ Standardization and technology will come at huge financial cost, which is unlikely to be met by a cashstrapped health service during a pandemic. In the United Kingdom, a more realistic solution utilizing existing resources would entail the wide distribution of box trainers to trainees, with progress rigorously charted by teleconference with trainers and peers. This approach has been used effectively in medical education in the past. ${ }^{29}$

In conclusion, this study shows that the use of laparoscopic box trainers translates to enhanced performance of basic skills by novices. Formal tuition, supervised practice and feedback may confer additional benefits compared with self-directed learning and practice, although the current study did not demonstrate a statistically significant effect. Further studies are needed to explore whether significant benefits can be achieved if tuition, supervised practice and feedback are introduced over a longer period of time.

\section{Conflict of interest}

The authors have no conflict of interest.

\section{Acknowledgement}

This study was carried out at the Royal College of Surgeons during the 7th National Undergraduate Surgery and Trauma Conference on 5 March 2016. This conference was run by the 2015-2016 committee of the Edinburgh Student Surgical Society, none of whom have any formal affiliation with eoSurgical or any other manufacturer of laparoscopic training material. eoSim box trainers were supplied on loan without charge by eoSurgical. eoSurgical had no input in the study design, data analysis or manuscript preparation. This study was supported by the Royal College of Surgeons of Edinburgh. This research did not receive any specific grant from funding agencies in the public, commercial, or not-for-profit sectors.

\section{References}

1. Gough IR. The impact of reduced working hours on surgical training in Australia and New Zealand. Surgeon 2011; 9(Suppl 1): S8-S9. https://doi.org/10.1016/j.surge.2010.11.010.

2. Joint Committee on Surgical Training. Curriculum - Preview our plans for the new curriculum due from August 2020. 10 
June 2019. Available from: https://www.jcst.org/jcst-news/ 2019/06/10/preview-our-plans-for-the-new-curriculum-duefrom-august-2020/. Accessed 23 Jun 2020.

3. Sallinen V, Akl EA, You JJ, Agarwal A, Shoucair S, Vandvik $\mathrm{PO}$, et al. Meta-analysis of antibiotics versus appendicectomy for non-perforated acute appendicitis. Br J Surg 2016; 103(6): 656-667. https://doi.org/10.1002/bjs.10147.

4. COVIDSurg Collaborative. Global guidance for surgical care during the COVID-19 pandemic. Br J Surg 2020; 107: 10971103. https://doi.org/10.1002/bjs.11646.

5. Al-Jabir A, Kerwan A, Nicola M, Alsafi Z, Khan M, Sohrabi C, et al. Impact of the coronavirus (COVID-19) pandemic on surgical practice - part 1. Int J Surg 2020; 79: 168-179. https://doi.org/10.1016/j.ijsu.2020.05.022.

6. Royal College of Surgeons of England. Updated intercollegiate general surgery guidance on COVID-19. 2020. Available from: https://www.rcseng.ac.uk/coronavirus/joint-guidance-for-surge ons-v2/. Accessed on 30 Jun 2020.

7. Society of American Gastrointestinal and Endoscopic Surgeons. SAGES and EAES recommendations regarding surgical responses to COVID-19 crisis. 2020. Available from: https://www.sages.org/recommendations-surgical-responsecovid-19/. Accessed on 30 Jun 2020.

8. Dawe SR, Pena GN, Windsor JA, Broeders JAJL, Cregan PC, Hewett PJ, et al. Systematic review of skills transfer after surgical simulation-based training. Br J Surg 2014; 101: 10631076. https://doi.org/10.1002/bjs.9482.

9. Vanderbilt A, Grover AC, Pastis NJ. Feldman M, Granados DD, Murithi LK, et al. Randomized controlled trials: a systematic review of laparoscopic surgery and simulation-based training. Glob J Health Sci 2015; 2: 310-327. https://doi. org/10.5539/gjhs.v7n2p310.

10. Gravante G, Venditti D. A systematic review on low-cost box models to achieve basic and advanced laparoscopic skills during modern surgical training. Surg Laparosc Endosc Percutan Tech 2013; 2: 109-120. https://doi.org/10.1097/SLE. 0b013e3182827c29.

11. Healy NA, Chang KH, Conneely JB, Malone C, Kerin MJ. Impact of supervised training on the acquisition of simulated laparoscopic skills. Ann R Coll Surg Engl 2013; 95(6): 1-6. https://doi.org/10.1308/003588413X13643054410340.

12. Armstrong BA, Gordon L, Grantcharov TP, Palter VN. The importance of feedback for surgical teams during the COVID19 pandemic. Br J Surg 2020; 107(10): e410-e411. https://doi. org/10.1002/bjs.11853.

13. Trehan A, Barnett-Vanes A, Carty MJ, McCulloch P, Maruthappu M. The impact of feedback of intraoperative technical performance in surgery: a systematic review. BMJ Open 2015; 5(6): e006759. https://doi.org/10.1136/bmjopen2014-006759.
14. Vassiliou MC, Feldman LS, Andrew CG, Bergman S, Leffondré, Stanbridge D, Fried GM. A global assessment tool for evaluation of intraoperative laparoscopic skills. Am J Surg 2005; 190: 107-113. https://doi.org/10.1016/j.amjsurg. 2005.04.004.

15. Hennessey IA, Hewett P. Construct, concurrent, and content validity of the eoSim laparoscopic simulator. J Laparoendosc Adv Surg Tech 2013; 23(10): 855-860. https://doi.org/10. 1089/lap.2013.0229.

16. Nagendran M, Troon CD, Davidson BR, Gurusamy KS. Laparoscopic surgical box model training for surgical trainees with no prior laparoscopic experience. Cochrane Database Syst Rev 2014; (1): CD010479. https://doi.org/10.1002/ 14651858.CD010479.pub2.

17. Crochet P, Schmitt A, Rambeaud C, Estrada JP, Karsenty G, Torre A, et al. Mandatory completion of a box trainer curriculum prior to laparoscopic apprenticeship in the OR for surgical residents: a before and after study. J Gynecol Obstet Hum Reprod 2018; 47(4): 157-161. https://doi.org/10.1016/j. jogoh.2018.01.002.

18. Halvorsen FH, Fosse E, Mjaland O. Unsupervised virtual reality training may not increase laparoscopic suturing skills. Surg Laparosc Endosc Percutan Tech 2011; 21: 458-461. http://doi.org/10.1097/SLE.0b013e31823bdf0c.

19. Xeroulis GJ, Park J, Moulton CA, Reznick RK, Leblanc, Dubrowski A. Teaching suturing and knot-typing skills to medical students: a randomized controlled study comparing computer-based video instruction and (concurrent and summary) expert feedback. Surgery 2007; 141(4): 442-449. https://doi.org/10.1016/j.surg.2006.09.012.

20. Ijgosse WM, Kengen B, van Goor H, Luursema JM. Peers versus pros: feedback using standards in simulation training. Am J Surg 2018; 216(6): 1223-1229. https://doi.org/10.1016/j. amjsurg. 2018.07.046.

21. O’Connor A, Schwaitzberg SD, Cao CG. How much feedback is necessary for learning to suture? Surg Endosc 2008; 22(7): 1614-1619. https://doi.org/10.1007/s00464-007-9645-6.

22. Van Bruwaene S, De Win G, Miserez M. How much do we need experts during laparoscopic suturing training. Surg Endosc 2009; 23(12): 2755-2761. https://doi.org/10.1007/ s00464-009-0498-z.

23. Moulton CA, Dubroski A, Macrae H, Graham B, Grober E, Reznick R. Teaching surgical skills: what kind of practice makes perfect: a randomized controlled trial. Ann Surg 2006; 244(3): 400-409. https://doi.org/10.1097/01.sla.000023 4808.85789.6a.

24. Mackay S, Morgan P, Datta V, Chang A, Darzi A. Practice distribution in procedural skills training: a randomized controlled trial. Surg Endosc 2002; 16(6): 957-961. https://doi10. 1007/s00464-001-9132-4. 
25. Verdaasdonk EG, Stassen LP, van Wijk RP, Dankelman J. The influence of different training schedules on the learning of psychomotor skills for endoscopic surgery. Surg Endosc 2007; 21(12): 214-219. https://doi10.1007/s00464-005-0852-8.

26. Bogdanova R, Boulanger P, Zheng B. Depth perception of surgeons in minimally invasive surgery. Surg Innov 2016; 23(5): 515-524. https://doi.org/10.1177/1553350616639141.

27. Fundamentals in Laparoscopic Surgery. SAGES. Available from: https://www.sages.org/projects/fls/ Accessed on 16 Mar 2021.
28. Hashimoto DA, Rosman G, Rus D, Meireles OR. Artificial intelligence in surgery: promises and perils. Ann Surg 2018; 268(1): 70-76. https://doi.org/10.1097/SLA.00000 00000002693.

29. Ehrlich H, McKenney M, Elkbuli A. We asked the experts: virtual learning in surgical education during the COVID-19 pandemic - shaping the future of surgical education and training. World J Surg 2020; 44(7): 2053-2055. https://doi.org/10. 1007/s00268-020-05574-3. 